\title{
Randomized Phase II Study of Pemetrexed Versus Gefitinib in Previously Treated Patients with Advanced Non-small Cell Lung Cancer
}

\author{
Young Saing Kim, $\mathrm{MD}^{1}$ \\ Eun Kyung Cho, MD, PhD ${ }^{1}$ \\ Hyun Sun Woo, MD ${ }^{1}$ \\ Junshik Hong, MD ${ }^{1}$ \\ Hee Kyung Ahn, MD ${ }^{1}$ \\ Inkeun Park, MD ${ }^{1}$ \\ Sun Jin Sym, MD, PhD ${ }^{1}$ \\ Sun Young Kyung, MD, PhD² \\ Shin Myung Kang, MD² \\ Jeong-Woong Park, MD, PhD² \\ Sung Hwan Jeong, MD, PhD² \\ Jinny Park, MD, PhD' \\ Jae Hoon Lee, MD, $P h D^{1}$ \\ Dong Bok Shin, MD, PhD ${ }^{1}$
}

Divisions of ${ }^{1}$ Hematology and Oncology and ${ }^{2}$ Pulmonary and Critical Care Medicine, Department of Internal Medicine, Gachon University Gil Medical Center, Incheon, Korea

Correspondence: Eun Kyung Cho, MD, PhD Division of Hematology and Oncology, Department of Internal Medicine,

Gachon University Gil Medical Center,

21 Namdong-daero 774beon-gil, Namdong-gu, Incheon 21565, Korea

Tel: 82-32-460-8314

Fax: 82-32-460-1754

E-mail: ekcho@gilhospital.com

Received October 28, 2014

Accepted December 19, 2014

Published Online March 2, 2015

\section{Purpose}

This study aimed to evaluate the efficacy and safety of pemetrexed versus gefitinib in patients with advanced non-small cell lung cancer (NSCLC) previously treated with chemotherapy.

\section{Materials and Methods}

Patients with advanced (stage IIIB or IV) or recurrent NSCLC were randomly assigned to receive either $500 \mathrm{mg} / \mathrm{m}^{2}$ of pemetrexed intravenously every 3 weeks or gefitinib 250 $\mathrm{mg} /$ day orally. The primary end point was progression-free survival (PFS) at 6 months.

\section{Results}

A total of 95 patients were enrolled ( 47 for pemetrexed and 48 for gefitinib). Most patients were male (72\%) and current/ex-smokers (69\%), and $80 \%$ had non-squamous cell carcinoma. The epidermal growth factor receptor (EGFR) mutation status was determined in 38 patients (40\%); one patient per each arm was positive for EGFR mutation. The 6-month PFS rates were $22 \%$ and $15 \%$ for pemetrexed and gefitinib, respectively $(p=0.35)$. Both arms showed an identical median PFS of 2.0 months and a median overall survival (OS) of 8.5 months. In EGFR wild-type patients, higher response rate (RR) and longer PFS as well as OS were achieved via pemetrexed compared with gefitinib, although there were no significant differences (RR: 39\% vs. 9\%, $p=0.07$; median PFS: 6.6 months vs. 3.1 months, $p=0.45$; median OS: 29.6 months vs. 12.9 months, $p=0.62$ ). Toxicities were mild in both treatment arms. Frequently reported toxicities were anemia and fatigue for pemetrexed, and skin rash and anorexia for gefitinib.

\section{Conclusion}

Both pemetrexed and gefitinib had similar efficacy with good tolerability as second-line treatment in unselected patients with advanced NSCLC. However, pemetrexed is considered more effective than gefitinib for EGFR wild-type patients.

Key words

Pemetrexed, Gefitinib, Non-small cell lung cancer, Epidermal growth factor receptor, Second-line

\section{Introduction}

Lung cancer is the leading cause of cancer death in Korea [1] and the United States [2]. Non-small cell lung cancer (NSCLC) accounts for approximately $85 \%$ of all lung cancers [3]. Approximately half of NSCLC patients are initially diag- nosed with advanced disease. For patients with negative or unknown driver mutation status, platinum-based combination chemotherapy represents the standard of care in advanced NSCLC [4]. However, almost all patients eventually develop progressive disease that requires further treatments after the initial therapy.

Recently, several therapeutic agents have shown efficacy 
in the second-line setting, and second-line treatment is now accepted as the standard of care [5]. Two cytotoxic agents, docetaxel and pemetrexed, and two epidermal growth factor receptor (EGFR) tyrosine kinase inhibitors (TKIs), erlotinib and gefitinib, are the standard options for patients whose disease has progressed during or after the first-line, platinum-based therapy. Docetaxel was first approved as a second-line therapy based on two randomized phase III trials $[6,7]$. Docetaxel showed longer survival and better quality of life compared with best supportive care (BSC) alone and with vinorebine or ifosfamide. Thereafter, similar median survival was observed for pemetrexed, but with less toxicity when compared to docetaxel [8]. Gefitinib also has a benefit for second-line treatment. Although the ISEL study [9] failed to demonstrate a survival benefit of gefitinib over BSC, subsequent phase III studies $[10,11]$ reported similar efficacy but better tolerability for gefitinib compared to docetaxel.

Both pemetrexed and gefitinib appear to have similar efficacies, but have different toxicity profiles and administration routes. This randomized phase II study was conducted in order to evaluate the efficacy and safety of pemetrexed and gefitinib in previously treated patients with advanced NSCLC.

\section{Materials and Methods}

\section{Patient eligibility}

The criteria for enrollment included the following: histologically- or cytologically-proven advanced (stage IIIB or IV) or recurrent NSCLC; disease progression after first-line or second-line chemotherapy; age $\geq 18$ years; Eastern Cooperative Oncology Group (ECOG) performance status (PS) $\leq 2$; at least one measurable lesion; adequate bone marrow (absolute neutrophil count $\geq 1,500 / \mathrm{mL}$ and platelet count $\geq 100,000 / \mathrm{mL}$ ), normal hepatic (bilirubin $\leq 1.25$ upper limit of normal [ULN] and hepatic transaminase $\leq 2.5 \mathrm{ULN}$ ), and renal (serum creatinine $<1.5 \mathrm{mg} / \mathrm{dL}$ ) functions; and an estimated life expectancy of at least 3 months. Patients with brain metastasis were eligible if treated with radiation therapy and clinically stable. Patients with chronic diarrhea of any grade, inflammatory bowel disease, uncontrolled comorbid illness, or other malignancies were excluded. During the study, there were protocol amendments that changed the entry criteria; patients who had squamous cell histology or activating EGFR mutations were excluded since August 2009. The protocol was reviewed and approved by the Gachon University Gil Medical Center (Incheon, Korea) Institutional Review Board. Written informed consent was obtained from all patients. This study is registered at ClinicalTrials.gov (NCT01783834).

\section{Treatment}

This study was a single-center, randomized, open-label, phase II trial. Patients were randomized to receive either pemetrexed or gefitinib, and were stratified according to histology (adenocarcinoma vs. others), smoking history (current/ ex-smoker vs. never-smoker), and PS (0 to 1 vs. 2). Pemetrexed $500 \mathrm{mg} / \mathrm{m}^{2}$ was administered intravenously over 10 minutes on day 1 of every 21-day cycle. Gefitinib $250 \mathrm{mg}$ was administered orally once daily (1 cycle for 21 days). Cycles were repeated until disease progression, unacceptable toxicity, or until the patient declined further treatment. Patients in the pemetrexed arm group were instructed to take folic acid ( $1 \mathrm{mg}$ orally daily) from 1 week before the administration of the first dose of pemetrexed, until 3 weeks after the last dose. Intramuscular injection of vitamin $B_{12}$ $(1.000 \mu \mathrm{g})$ was administered 1 week before the first dose administration of pemetrexed and was repeated every three cycles.

\section{Dose modification}

Dose adjustments to new cycles were based on the consideration of the worst toxicity observed during the previous cycle. Treatment interruptions up to 14 days were allowed for recovery from adverse events. Pemetrexed treatment was started only when the neutrophil count was $\geq 1,000 / \mathrm{mL}$, the platelet count was $\geq 75,000 / \mathrm{mL}$, and non-hematologic toxicities were grade $\leq 1$. Pemetrexed doses were reduced by $25 \%$ in patients with grade $\geq 4$ neutropenia, febrile neutropenia, grade $\geq 3$ thrombocytopenia, or a grade $\geq 3$ non-hematologic toxicity, other than nausea, vomiting, and alopecia. Patients who experienced grade $\geq 3$ hypersensitivity reactions or required two successive dose reductions were withdrawn from the study. Gefitinib administration was interrupted when patients developed grade $\geq 3$ diarrhea or grade $\geq 3$ skin toxicities. Gefitinib was permanently discontinued for patients who developed interstitial lung disease.

\section{Evaluation}

The baseline evaluation included detailed medical history, physical examination, complete blood counts, blood chemistries, and imaging for tumor assessment. Patients were assessed with medical history, physical examination, complete blood counts, blood chemistries, and X-rays before each treatment cycle. Tumor responses were classified according to the Response Evaluation Criteria in Solid Tumors guidelines ver. 1.1. The response was evaluated 
based on the findings from the computed tomography scan of the chest and upper abdomen. Clinical responses were assessed every two cycles or earlier in the case of clinical deterioration. The toxic effects were assessed according to the National Cancer Institute Common Toxicity Criteria ver. 3.0. For EGFR gene analysis, genomic DNA was extracted from paraffin-embedded tumor tissues and amplified using polymerase chain reaction. EGFR mutational status was examined in exons 18 to 21 via direct sequencing.

\section{Statistical analysis}

The primary end point of this study was progression-free survival (PFS) rate at 6 months, and secondary end-points were PFS, toxicity, response rate (RR), and overall survival (OS). In order to estimate the activity and safety of the two arms simultaneously and to minimize patient selection bias, this study was conducted using a randomized, non-comparative phase II design. We calculated the sample size separately for each arm based on Fleming's single-stage design. Assuming an expected 6-month PFS rate of 25\% and a threshold rate of $10 \%, 49$ patients per arm were required in order to yield $90 \%$ power at the type I error rate of $5 \%$. Timeto-event data were analyzed using the Kaplan-Meier method. Estimation of the 6-month PFS rate was based on the proportion of patients who were alive without documented disease progression at 6 months after the treatment start date. Exploratory analyses were planned for comparison of clinical outcomes between the two arms. Categorical variables were compared using Pearson chi-square test or Fisher exact test. The log-rank test was used for the evaluation of survival differences between the two arms. Cox proportional hazard method was used for identification of independent prognostic factors of survival. Statistical significance was accepted for $p$-values of $<0.05$. All analyses were performed using SPSS ver. 19.0 (SPSS Inc., Chicago, IL).

\section{Results}

\section{Patient characteristics}

A total of 95 patients were randomly assigned to the pemetrexed arm $(n=47)$ or the gefitinib arm $(n=48)$, between December 2007 and June 2013. The study was closed early due to poor accrual after pemetrexed/ cisplatin, and gefitinib were approved as the first-line treatment in Korea. A summary of patient baseline characteristics is shown in Table 1. According to patient characteristics, $72 \%$ were male, $20 \%$ had squamous cell carcinoma, and $98 \%$ had unknown or wild-type EGFR status. The treatment groups were generally well balanced for baseline characteristics, except that the gefitinib group included a higher percentage of patients with pleural metastasis (54\% vs. 34\%), EGFR wild-type patients ( $48 \%$ vs. $28 \%$ ), and patients with no response to previous chemotherapy (46\% vs. 32\%) than the pemetrexed group.

\section{Efficacy}

After a median follow-up period of 60.6 months, 45 patients in the pemetrexed arm and 48 patients in the gefitinib arm showed progression. The 6-month PFS rates were $22 \%$ (95\% confidential interval [CI], $10 \%$ to $34 \%$ ) in the pemetrexed arm and $15 \%$ (95\% CI, $5 \%$ to $25 \%)$ in the gefitinib arm. The median PFS was 2.0 months (95\% CI, 1.4 to 2.7 months) and 2.0 months (95\% CI, 1.2 to 2.8 months) in the pemetrexed arm and the gefitinib arm, respectively (Fig. 1A). Results of exploratory analyses showed no significant differences in the 6-month PFS rate $(\mathrm{p}=0.35)$ and PFS $(\mathrm{p}=0.71)$ between the two arms.

Salvage treatment was administered to 33 patients $(70 \%)$ and 32 patients $(67 \%)$ in the pemetrexed arm and the gefitinib arm, respectively. Thirty patients $(64 \%)$ in the pemetrexed arm received gefitinib or erlotinib for subsequent therapy, and 23 patients (48\%) in the gefitinib arm were crossed over to pemetrexed. At the time of analysis, 42 patients (89\%) in the pemetrexed arm and 43 patients (90\%) in the gefitinib arm had died. An identical median OS of 8.5 months was achieved for both arms, and 1-year survival rates were $34 \%$ for pemetrexed and $41 \%$ for gefitinib (Fig. 1B). For all patients, the results of a multivariable analysis showed that ECOG PS 0 to 1 (hazard ratio [HR], 0.43; 95\% $\mathrm{CI}, 0.26$ to 0.71 ) and adenocarcinoma (HR, $0.59 ; 95 \% \mathrm{CI}, 0.35$ to 0.98 ) were independent prognostic factors associated with longer OS.

The response could not be assessed in four patients; two in the pemetexed arm (patient refusal after the first cycle and follow-up loss) and two in the gefitinib arm (patient refusal after the first cycle and treatment-related death). According to the intent-to-treat principle, the objective RRs were $13 \%$ in the pemetrexed group and $8 \%$ in the gefitinib group $(\mathrm{p}=0.52)$. The disease control rates for pemetrexed and gefitinib were $45 \%$ and $35 \%$, respectively $(\mathrm{p}=0.36)$.

\section{Efficacy analysis according to EGFR mutation status}

EGFR mutation status was known in 38 patients (40\%). EGFR mutation was detected in one patient per each group. The patient in the pemetrexed group had deletion in exon 19 and received subsequent gefitinib, and the OS was 35.4 months. A patient in the gefitinib group with G719X mutation showed an OS of 8.5 months. In 36 patients with wild- 
Table 1. Baseline patient characteristics

\begin{tabular}{|c|c|c|c|}
\hline Characteristic & Pemetrexed (n=47) & Gefitinib $(n=48)$ & p-value \\
\hline \multicolumn{4}{|l|}{ Sex } \\
\hline Male & $33(70)$ & $35(73)$ & \multirow[t]{2}{*}{0.77} \\
\hline Female & $14(30)$ & $13(27)$ & \\
\hline \multicolumn{4}{|l|}{ Age (yr) } \\
\hline Median (range) & $64(31-81)$ & $67(42-82)$ & \multirow[t]{2}{*}{0.26} \\
\hline$\geq 65$ & $21(45)$ & $27(56)$ & \\
\hline \multicolumn{4}{|l|}{ Performance status } \\
\hline 0 & $5(11)$ & $5(10)$ & \multirow[t]{3}{*}{0.94} \\
\hline 1 & $27(57)$ & $26(54)$ & \\
\hline 2 & $15(32)$ & $17(35)$ & \\
\hline \multicolumn{4}{|l|}{ Smoking status } \\
\hline Current or ex-smoker & $33(70)$ & $33(69)$ & \multirow[t]{2}{*}{0.88} \\
\hline Never-smoker & $14(30)$ & $15(31)$ & \\
\hline \multicolumn{4}{|l|}{ Pathologic subtype } \\
\hline Adenocarcinoma & $29(62)$ & $31(65)$ & \multirow[t]{4}{*}{0.99} \\
\hline Squamous cell carcinoma & $10(21)$ & $9(19)$ & \\
\hline LCNEC & $1(2)$ & $1(2)$ & \\
\hline NSCLC not otherwise specified & $7(15)$ & $7(15)$ & \\
\hline \multicolumn{4}{|l|}{ Stage at treatment } \\
\hline IIIB & $3(6)$ & $2(4)$ & \multirow[t]{2}{*}{$0.68^{\mathrm{a})}$} \\
\hline $\mathrm{IV}^{\mathrm{b})}$ & $44(94)$ & $46(96)$ & \\
\hline \multicolumn{4}{|l|}{ Metastatic sites } \\
\hline Lung to lung & $18(38)$ & $21(44)$ & 0.59 \\
\hline Pleura & $16(34)$ & $26(54)$ & 0.05 \\
\hline Brain & $7(15)$ & $4(8)$ & 0.32 \\
\hline$\geq 2$ sites & $18(38)$ & $23(48)$ & 0.34 \\
\hline \multicolumn{4}{|l|}{ Treatment sequence } \\
\hline 2nd-line & $32(68)$ & $30(63)$ & \multirow[t]{2}{*}{0.57} \\
\hline 3rd-line & $15(32)$ & $18(38)$ & \\
\hline \multicolumn{4}{|l|}{ Previous chemotherapy } \\
\hline Platinum-based combinations & $41(87)$ & $40(83)$ & \multirow[t]{3}{*}{0.80} \\
\hline Non-platinum combinations & $3(6)$ & $4(8)$ & \\
\hline Monotherapy without platinum & $3(6)$ & $4(8)$ & \\
\hline \multicolumn{4}{|c|}{ Best response, any prior chemotherapy } \\
\hline Complete response & 0 & $1(2)$ & \multirow[t]{4}{*}{0.23} \\
\hline Partial response & $32(68)$ & $25(52)$ & \\
\hline Stable disease & $12(26)$ & $13(29)$ & \\
\hline Progressive disease & $3(6)$ & $8(17)$ & \\
\hline \multicolumn{4}{|l|}{ EGFR mutation } \\
\hline Mutant & $1(2)$ & $1(2)$ & \multirow[t]{3}{*}{0.12} \\
\hline Wild-type & $13(28)$ & $23(48)$ & \\
\hline Unknown & $33(70)$ & $24(50)$ & \\
\hline
\end{tabular}

Values are presented as number (\%). LCNEC, large-cell neuroendocrine carcinoma; NSCLC, non-small cell lung cancer; EGFR, epidermal growth factor receptor. ${ }^{a)}$ Fisher exact test, ${ }^{\mathrm{b}}$ Includes four patients with recurrent disease (one patient for pemetrexed and three patients for gefitinib).

type EGFR, a trend for better RR was observed in the pemetrexed group compared to the gefitinib group (39\% vs. 9\%, $\mathrm{p}=0.07)$; the median PFS and median OS were longer in the pemetrexed arm than in the gefitinib arm, but the differences were not statistically significant (median PFS: 6.6 months vs. 3.1 months, $\mathrm{p}=0.45$; median OS: 29.6 months vs. 12.9 months, 

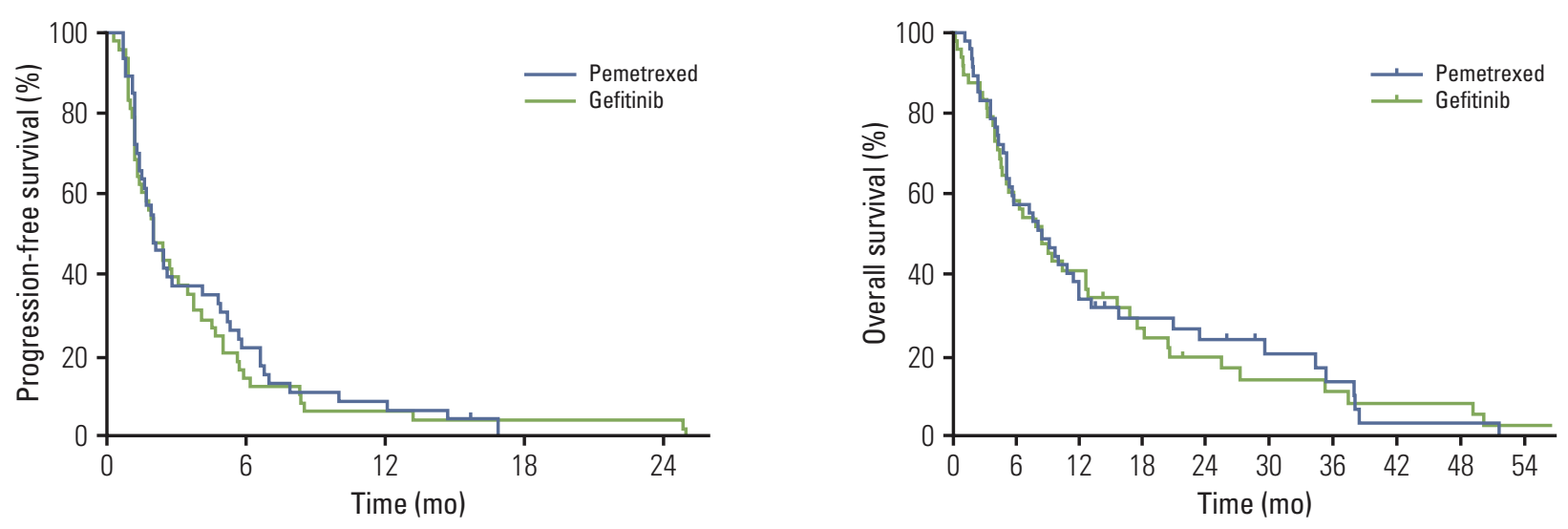

Fig. 1. Kaplan-Meier curves for progression-free survival (A) and overall survival (B).

$\mathrm{p}=0.62)$. In patients with unknown EGFR mutation status, similar efficacy outcomes were observed between pemetrexed and gefitinib (median PFS: 2.0 months vs. 1.5 months, $\mathrm{p}=0.55$; median OS: 7.7 months vs. 6.4 months, $\mathrm{p}=0.80$; RR: $0 \%$ vs. $8 \%, \mathrm{p}=0.17)$.

\section{Efficacy analysis according to histologic subtypes}

When the analysis was limited to patients with non-squamous carcinoma, the median PFS was 2.1 months $(95 \% \mathrm{CI}$, 1.6 to 2.5 months) and 2.0 months (95\% CI, 0.8 to 3.1 months) for the pemetrexed and gefitinib arms, respectively $(\mathrm{p}=0.66)$, with the median OS of 9.8 months $(95 \%$ CI, 5.3 to 14.3 months) and 9.5 months ( $95 \%$ CI, 3.9 to 15.1 months), respectively ( $\mathrm{p}=0.84$ ). The objective RRs were also similar in both arms ( $16 \%$ vs. $10 \%, \mathrm{p}=0.53)$. For the subpopulation comprising squamous cell carcinoma, there was no significant difference between the pemetrexed and gefitinib arms regarding PFS (median, 1.7 months vs. 2.7 months, $\mathrm{p}=0.73$ ), OS (median, 4.9 months vs. 5.4 months, $\mathrm{p}=0.84)$, and RR ( $0 \%$ in both arms).

\section{Toxicity}

All patients were assessed for toxicity. The median number of cycles administered was three for both arms (range, 1 to 23 for the pemetrexed arm and 1 to 35 for the gefitinib arm). The reasons for treatment discontinuation were disease progression ( $87 \%$ for pemetrexed and $88 \%$ for gefitinib), adverse events (9\% and 10\%), and follow-up loss (2\% and $0 \%$ ). Treatment delays or interruptions due to adverse events were required in eight patients (15\%) and four patients $(8 \%)$ in the pemetrexed arm and the gefitinib arm, respectively.
The dose of pemetrexed was decreased in two patients (4\%).

A list of treatment-related hematologic and non-hematologic toxicities is shown in Table 2. The most common adverse events were anemia $(51 \%)$ and fatigue $(45 \%)$ with pemetrexed, and skin rash (46\%) and anorexia (42\%) with gefitinib. Grade 3 or 4 adverse events occurred in $21 \%$ of patients in the pemetrexed arm group and 19\% in the gefitinib arm group. There was one treatment-related deaths resulting from pneumonia in each arm. Interstitial lung disease was noted in two patients who received gefitinib.

\section{Discussion}

This study was a prospective, randomized phase II study to evaluate the efficacy and safety of pemetrexed or gefitinib in previously treated advanced NSCLC. Both regimens demonstrated modest activity as the second-line treatment with a median PFS of 2.0 months and a median OS of 8.5 months in each arm. Toxicities related to both regimens were mild and well-tolerated. The results of exploratory analyses for comparison of clinical outcomes between the two arms showed no significant difference in efficacy between pemetrexed and gefitinib in the overall population and in patients with EGFR wild-type tumors.

For patients with EGFR activating mutations, EGFR TKIs are the favored second-line therapy if not used in the firstline setting $[12,13]$. However, the role of EGFR TKIs as the second-line treatment for EGFR wild-type or EGFR unknown patients remains controversial. Both pemetrexed and gefitinib are currently used in East Asia as the standard 
Table 2. Adverse events

\begin{tabular}{|c|c|c|c|c|}
\hline \multirow{2}{*}{ Variable } & \multicolumn{2}{|c|}{ Pemtrexed $(n=47)$} & \multicolumn{2}{|c|}{ Gefitinib $(n=48)$} \\
\hline & All grades & Grade 3 to 4 & All grades & Grade 3 to 4 \\
\hline \multicolumn{5}{|l|}{ Hematologic toxicity } \\
\hline Anemia & $24(51)$ & $5(11)$ & $10(21)$ & $1(2)$ \\
\hline Leukocytopenia & $2(4)$ & - & - & - \\
\hline Neutropenia & $3(6)$ & - & - & - \\
\hline Thrombocytopenia & $3(6)$ & $1(2)$ & - & - \\
\hline \multicolumn{5}{|l|}{ Non-hematologic toxicity } \\
\hline Skin rash & $5(11)$ & - & $22(46)$ & - \\
\hline Fatigue & $21(45)$ & $2(4)$ & $10(21)$ & $1(2)$ \\
\hline Anorexia & $19(40)$ & $2(4)$ & $20(42)$ & - \\
\hline Nausea & $10(21)$ & $1(2)$ & $12(25)$ & $1(2)$ \\
\hline Vomiting & $4(9)$ & - & $7(15)$ & $1(2)$ \\
\hline Stomatitis & $4(9)$ & - & $9(19)$ & - \\
\hline Constipation & $10(21)$ & - & $1(2)$ & - \\
\hline Diarrhea & $4(9)$ & - & $8(17)$ & $2(4)$ \\
\hline Infection & $7(15)$ & $3(6)$ & $4(8)$ & $2(4)$ \\
\hline Edema & $2(4)$ & - & $2(4)$ & - \\
\hline Interstitial lung disease & - & - & $2(4)$ & $1(2)$ \\
\hline
\end{tabular}

Values are presented as number $(\%)$.

second-line treatment for advanced non-squamous NSCLC. Previously, two randomized trials compared pemetrexed with gefitinib in a second-line treatment setting. The aim of the phase III KCSG-LU08-01 study [14] $(n=135)$ was to compare gefitinib with pemetrexed in a clinically selected population (never smoker Korean patients with pulmonary adenocarcinoma). The results clearly favored gefitinib treatment; the median PFS (9.0 months vs. 3.0 months, $\mathrm{p}=0.0006$ ) and objective RR (59\% vs. $22 \%, \mathrm{p}=0.001)$ were significantly increased with gefitinib, although there was no significant difference in OS (22.2 months vs. 18.9 months, $\mathrm{p}=0.37)$. A recent Chinese phase II study (CTONG0806) reported that pemetrexed was superior to gefitinib in a second-line setting for EGFR wild-type non-squamous NSCLC [15]. Final updated results presented at the World Conference on Lung Cancer 2013 demonstrated a significant improvement in PFS (4.9 months vs. 1.6 months, $\mathrm{p}<0.001$ ), disease control rate $(60.5 \%$ vs. $29.6 \%, \mathrm{p}<0.001)$, and a trend of favorable OS (12.4 months vs. 9.6 months, $\mathrm{p}=0.077$ ) with pemetrexed compared to gefitinib. In contrast to the previous studies, similar efficacy between pemetrexed and gefitinib in our study might be derived from the enrollment of a clinically unselected population. Our study included a high proportion of males, current or ex-smokers, and patients with unknown EGFR status. In addition, $20 \%$ of patients had squamous cell tumors. Likewise, previous phase III trials [10,11,16,17] conducted in unselected patients demonstrated equivalent efficacy between EGFR TKIs, compared to single-agent chemotherapy, including pemetrexed or docetaxel in the second-line setting; EGFR TKI therapy yielded a RR of approximately $8 \%$ to $9 \%$, a median PFS of 1.5 to 3.6 months, and a median OS of 5.3 to 11.5 months in EGFR-unselected advanced NSCLC.

There is growing evidence indicating that, in patients with EGFR wild-type disease, single agent chemotherapy is superior to EGFR TKIs for the second-line or subsequent treatment. The TAILOR study [18] was specifically designed for comparison of erlotinib with docetaxel only in wild-type EGFR tumors. Significantly better PFS and OS were observed with docetaxel chemotherapy compared with erlotinib (HR for PFS: 0.71 ; 95\% CI, 0.53 to 0.95; HR for OS: 0.73; 95\% CI, 0.53 to 1.00). Subsequently, the DELTA trial [19] also reported longer PFS favoring docetaxel over erlotinib in a subgroup analysis of 199 EGFR wild-type patients (median PFS 2.9 months vs. 1.3 months; HR, $0.64 ; 95 \%$ CI, 0.47 to 0.85 ). In addition, a recent meta-analysis concluded that EGFR TKIs was statistically inferior to chemotherapy in delaying disease progression for EGFR wild-type patients who were suitable to receive chemotherapy (HR, 1.23; 95\% CI, 1.05 to 1.46) [13]. These findings suggest that the exact mutational status should be determined in order to guide rational decision making for the second-line treatment. In the current study, no significant difference of efficacy was observed between pemetrexed and gefitinib among EGFR wild-type 
patients. This observation may be explained by a small number of patients in our study who were confirmed to have $E G F R$ wild-type tumors. In addition, we used direct sequencing for EGFR mutation analysis. The relative low sensitivity of direct sequencing tends to cause false-negative results. Actually, in the gefitinib arm, three patients found to be EGFR mutation-negative showed satisfactory clinical outcome with gefitinib (PFS of 13.2 to 25.0 months). Improvement of tissue acquisition and the application of more sensitive genotype assays are needed in order to clearly define the mutational status in patients with advanced NSCLC.

Pemetrexed is more effective than docetaxel or gemcitabine as a treatment for patients with non-squamous NSCLC, with an overall favorable toxicity profile $[20,21]$. Pemetrexed also has an established role in maintenance therapy for advanced NSCLC $[22,23]$. Therefore, pemetrexed is increasingly used whether as part of the first-line platinumbased doublet or as maintenance therapy, and its availability in the second-line setting has decreased for patients with non-squamous histology. In daily clinical practice, docetaxel and EGFR TKIs are usually the remaining options as the second-line treatment. The goal of second-line treatment in advanced NSCLC is palliation. For the optimal second-line treatment, patient-related factors should be considered, including their priorities, PS, and comorbidities. Based on this background, EGFR TKIs are still an important option for patients with unknown EGFR status, or for patients with wild-type EGFR who are unfit to receive docetaxel chemotherapy, because of equivalent efficacy in unselected patients and mild toxicity profile.

Our study has some limitations. First, it was conducted at a single institution, in a small study population using an initial non-comparative design, which reduced the accuracies of comparison between the two treatment arms. Second, our study was initiated before the interaction between peme- trexed efficacy and histology was detected; thus, patients with squamous cell carcinoma (20\%) were included in the analyses, which could have biased the results. However, the results of a subgroup analysis of non-squamous patients showed no significant differences in PFS $(\mathrm{p}=0.66)$ and OS ( $\mathrm{p}=0.84$ ) between the two arms. Third, the EGFR mutation status was not determined in more than half of patients, and there was an imbalance in the distribution of EGFR mutation status between the two arms. This finding is explained by the fact that gefitinib was usually used without EGFR mutation analysis in clinical practice when this study was initiated, and EGFR mutation testing was not feasible in some patients due to insufficient or inadequate tissue samples. However, our results reflect a daily clinical situation in that the decision making should be done without knowledge of genotype information.

\section{Conclusion}

The present study showed that both pemetrexed and gefitinib had similar efficacy with good tolerability as the second-line treatment in unselected patients with advanced NSCLC. However, pemetrexed is considered more effective than gefitinib for the second-line treatment in patients with EGFR wild-type disease.

\section{Conflicts of Interest}

Conflict of interest relevant to this article was not reported.

\section{References}

1. Jung KW, Won YJ, Kong HJ, Oh CM, Seo HG, Lee JS. Cancer statistics in Korea: incidence, mortality, survival and prevalence in 2010. Cancer Res Treat. 2013;45:1-14.

2. Siegel R, Naishadham D, Jemal A. Cancer statistics, 2013. CA Cancer J Clin. 2013;63:11-30.

3. Govindan R, Page N, Morgensztern D, Read W, Tierney R, Vlahiotis A, et al. Changing epidemiology of small-cell lung cancer in the United States over the last 30 years: analysis of the surveillance, epidemiologic, and end results database. J Clin Oncol. 2006;24:4539-44.

4. Azzoli CG, Temin S, Aliff T, Baker S Jr, Brahmer J, Johnson DH, et al. 2011 Focused update of 2009 American Society of
Clinical Oncology Clinical Practice Guideline update on chemotherapy for stage IV non-small-celllung cancer. J Clin Oncol. 2011;29:3825-31.

5. Azzoli CG, Baker S Jr, Temin S, Pao W, Aliff T, Brahmer J, et al. American Society of Clinical Oncology Clinical Practice Guideline update on chemotherapy for stage IV non-smallcell lung cancer. J Clin Oncol. 2009;27:6251-66.

6. Fossella FV, DeVore R, Kerr RN, Crawford J, Natale RR, Dunphy F, et al. Randomized phase III trial of docetaxel versus vinorelbine or ifosfamide in patients with advanced non-small-cell lung cancer previously treated with platinumcontaining chemotherapy regimens. The TAX 320 Non-Small 
Cell Lung Cancer Study Group. J Clin Oncol. 2000;18:2354-62.

7. Shepherd FA, Dancey J, Ramlau R, Mattson K, Gralla R, O'Rourke M, et al. Prospective randomized trial of docetaxel versus best supportive care in patients with non-small-cell lung cancer previously treated with platinum-based chemotherapy. J Clin Oncol. 2000;18:2095-103.

8. Hanna N, Shepherd FA, Fossella FV, Pereira JR, De Marinis $\mathrm{F}$, von Pawel J, et al. Randomized phase III trial of pemetrexed versus docetaxel in patients with non-small-cell lung cancer previously treated with chemotherapy. J Clin Oncol. 2004;22: 1589-97.

9. Thatcher N, Chang A, Parikh P, Rodrigues Pereira J, Ciuleanu $\mathrm{T}$, von Pawel J, et al. Gefitinib plus best supportive care in previously treated patients with refractory advanced non-small-cell lung cancer: results from a randomised, placebo-controlled, multicentre study (Iressa Survival Evaluation in Lung Cancer). Lancet. 2005;366:1527-37.

10. Kim ES, Hirsh V, Mok T, Socinski MA, Gervais R, Wu YL, et al. Gefitinib versus docetaxel in previously treated non-smallcell lung cancer (INTEREST): a randomised phase III trial. Lancet. 2008;372:1809-18.

11. Maruyama R, Nishiwaki Y, Tamura T, Yamamoto N, Tsuboi M, Nakagawa K, et al. Phase III study, V-15-32, of gefitinib versus docetaxel in previously treated Japanese patients with non-small-cell lung cancer. J Clin Oncol. 2008;26:4244-52.

12. Douillard JY, Shepherd FA, Hirsh V, Mok T, Socinski MA, Gervais R, et al. Molecular predictors of outcome with gefitinib and docetaxel in previously treated non-small-cell lung cancer: data from the randomized phase III INTEREST trial. J Clin Oncol. 2010;28:744-52.

13. Lee CK, Brown C, Gralla RJ, Hirsh V, Thongprasert S, Tsai $\mathrm{CM}$, et al. Impact of EGFR inhibitor in non-small cell lung cancer on progression-free and overall survival: a meta-analysis. J Natl Cancer Inst. 2013;105:595-605.

14. Sun JM, Lee KH, Kim SW, Lee DH, Min YJ, Yun HJ, et al. Gefitinib versus pemetrexed as second-line treatment in patients with nonsmall cell lung cancer previously treated with platinum-based chemotherapy (KCSG-LU08-01): an open-label, phase 3 trial. Cancer. 2012;118:6234-42.

15. Yang J, Cheng Y, Zhao M, Zhou Q, Yan HH, Zhang L, et al. A phase II trial comparing pemetrexed with gefitinib as the second-line treatment of nonsquamous NSCLC patients with wild-type EGFR (CTONG0806). In: 2013 ASCO Annual Meet- ing; 2013 May 31-Jun 4; Chicago, IL, USA. Alexandria, VA: American Society of Clinical Oncology; 2013. Abstr 8042.

16. Karampeazis A, Voutsina A, Souglakos J, Kentepozidis N, Giassas S, Christofillakis C, et al. Pemetrexed versus erlotinib in pretreated patients with advanced non-small cell lung cancer: a Hellenic Oncology Research Group (HORG) randomized phase 3 study. Cancer. 2013;119:2754-64.

17. Ciuleanu T, Stelmakh L, Cicenas S, Miliauskas S, Grigorescu AC, Hillenbach $C$, et al. Efficacy and safety of erlotinib versus chemotherapy in second-line treatment of patients with advanced, non-small-cell lung cancer with poor prognosis (TITAN): a randomised multicentre, open-label, phase 3 study. Lancet Oncol. 2012;13:300-8.

18. Garassino MC, Martelli O, Broggini M, Farina G, Veronese S, Rulli E, et al. Erlotinib versus docetaxel as second-line treatment of patients with advanced non-small-cell lung cancer and wild-type EGFR tumours (TAILOR): a randomised controlled trial. Lancet Oncol. 2013;14:981-8.

19. Kawaguchi T, Ando M, Asami K, Okano Y, Fukuda M, Nakagawa $\mathrm{H}$, et al. Randomized phase III trial of erlotinib versus docetaxel as second- or third-line therapy in patients with advanced non-small-cell lung cancer: Docetaxel and Erlotinib Lung Cancer Trial (DELTA). J Clin Oncol. 2014;32:1902-8.

20. Scagliotti G, Hanna N, Fossella F, Sugarman K, Blatter J, Peterson $\mathrm{P}$, et al. The differential efficacy of pemetrexed according to NSCLC histology: a review of two phase III studies. Oncologist. 2009;14:253-63.

21. Scagliotti GV, Parikh P, von Pawel J, Biesma B, Vansteenkiste J, Manegold C, et al. Phase III study comparing cisplatin plus gemcitabine with cisplatin plus pemetrexed in chemotherapynaive patients with advanced-stage non-small-cell lung cancer. J Clin Oncol. 2008;26:3543-51.

22. Paz-Ares LG, de Marinis F, Dediu M, Thomas M, Pujol JL, Bidoli $P$, et al. PARAMOUNT: final overall survival results of thephase III study of maintenance pemetrexed versus placebo immediately after induction treatment with pemetrexed plus cisplatin for advanced nonsquamous non-small-cell lung cancer. J Clin Oncol. 2013;31:2895-902.

23. Ciuleanu T, Brodowicz T, Zielinski C, Kim JH, Krzakowski M, Laack E, et al. Maintenance pemetrexed plus best supportive care versus placebo plus best supportive care for non-smallcell lung cancer: a randomised, double-blind, phase 3 study. Lancet. 2009;374:1432-40. 